\title{
Service-Based Business Network Modelling: Application to Dynamic Logistics
}

\author{
Alexander Smirnov and Nikolay Shilov \\ Institution of the Russian Academy of Sciences \\ St.Petersburg Institute for Informatics and Automation RAS (SPIIRAS), \\ 39, 14 Line, St. Petersburg, 19978, Russia \\ \{smir, oleg, nick\}@iias.spb.su
}

\begin{abstract}
Appearance of business networks can be considered as a response to changes in global markets. Networks can be seen as a step beyond the linear supply chain topography. The paper proposes an approach to business network modelling based on service networks. Since the centralized control is not always possible, presented approach proposes decentralized communication and ad-hoc decision making based on the current situation state and its possible future development. An approach presented is based on application of such technologies as ontology and context management to knowledge sharing. Ontologies are used for description of knowledge domains. The paper also presents experience of application of the above approach to the area of dynamic logistics. The considered problem takes into account continuously changing problem environment and requires nearly real-time solving.
\end{abstract}

Keywords: Service networks, business network modelling, ontology, context, dynamic logistics.

\section{Introduction}

Modern global companies have to build supply network strategies that provide maximum flexibility and can optimally respond to changes in their environment [1-3]. The emergence of business networks is one of the consequences of these changes. The automotive industry is a good example of this phenomenon. Today one of the most important competitive advantages for car makers is their ability to manufacture customised cars with a reduced lead time. At the same time, it is necessary to avoid significant inventory levels in order to keep costs lower. Such a strategy is called Build-to-Order (BTO) and stands for the capability to quickly build customized products upon receipt of customer orders without precise forecasts, inventory, or purchasing delays. In the BTO networks, customer orders are introduced in advance of, or at the start of the production process. An opposing strategy is build-to-stock (BTS), whereby the product is built prior to demand [4].

Complex decision making faces problems of management and sharing of huge amount of information \& knowledge from distributed and heterogeneous sources (experts, electronic documents, real-time sensors, etc.) belonging to business network members, personalization, availability of up-to-date and accurate information provided by the dynamic environment. The problems include search of right sources, extraction of content, presentation of results in a personalized way, and other. As a rule, the content of several sources has to be extracted and processed (e.g., fused, converted, 
checked) to produce required information. Due to such factors as different data formats, interaction protocols and others this leads to a problem of semantic interoperability.

Knowledge sharing and exchange in a business network are highly important and should be achieved at both technical and semantic levels. The interoperability at the technical level is addressed in a number of research efforts. It is usually represented by such approaches as e.g., SOA (service-oriented architecture) [5] and on the appropriate standards like WSDL and SOAP [6]. The semantic level of interoperability in the flexible supply network is also paid significant attention. As an example (probably the most widely known), the Semantic Web initiative is worth mentioning [7]. The main idea is to use ontologies for knowledge and terminology description.

The approach presented in this paper also relies on the ontological knowledge representation for its sharing. Ontologies are widely used for problem domain description in the modern information systems to support semantic interoperability. An ontology is an explicit specification of a structure of a certain domain. It includes a vocabulary for referring to notions of the subject area, and a set of logical statements expressing the constraints existing in the domain and restricting the interpretation of the vocabulary [8]. Ontologies support integration of resources that were developed using different vocabularies and different perspectives of the data. To achieve semantic interoperability, systems must be able to exchange data so that the precise meaning of the data is readily accessible and the data itself can be translated by any system into the form that it understands [9].

Ontologies facilitate information retrieval over collections of distributed and heterogeneous information sources; they help to provide for semantic integration of information and facilitate interoperability between heterogeneous knowledge sources at high level of abstraction [10]. The conceptual model of the proposed ontology-driven knowledge sharing is based on the earlier developed idea of knowledge logistics [11]. It correlates with the conceptual integration developed within the Athena project [12]. The ontology describes common entities of the enterprise systems and relationships between them. As a result, it is possible to treat all available knowledge and competencies as one distributed knowledge base.

Centralized control in complex distributed systems is not always possible: for example, business networks consist of independent companies and do not have a central decision making unit. Thus, decentralized organisation of distributed independent components is a promising architecture for such kind of systems [13-15]. However, in order for the self-organisation to operate it is necessary to solve a number of problems including: (i) registration and cancelling of registration of network elements, (ii) preparation of initial state, (iii) self-configuration: finding appropriate network elements [16], negotiation of conditions and assignment of links, and preparation of alternative configurations. Different research projects are devoted to self-management of such networks: self -contextualization, -optimization, -organization, -configuration, -adaptation, -healing, -protection [17].

The following major requirements to the approach to business network modelling have been selected (some of the decision making processes in business networks have been identified in [18]): (i) intensive information exchange, (ii) distributed architecture, (iii) decentralised control, (iv) semantic-based information processing, (v) ad-hoc decision making support based on the current situation state and its possible future development. The developed methodology proposes integration of environmental information in a certain context. The context is any information that can be used to characterize the situation of an entity where an entity is a person, place, or object that is considered 
relevant to the interaction between a user and an application, including the user and applications themselves [19]. The context is purposed to represent only relevant information from the large amount of those. Relevance of information is evaluated on a basis how they are related to a modelling of an ad-hoc problem. A number of already solved problems and problems to be solved includes interoperability at both technological and semantic level, situation understanding by the members via information exchange, protocols of ad-hoc decision making for self-organization. Proposed technological framework incorporates such technologies as situation management, knowledge and ontology management, profiling, Web-services, decision support, negotiation protocols.

The proposed methodology is based on the earlier developed concept of knowledge logistics [20] and includes such technologies as situation management, ontology management, profiling and intelligent agents [18]. Standards of information exchange (e.g., Web-service standards), negotiation protocols, decision making rules, etc. are used for information exchange and rapid establishing of ad-hoc partnerships and agreements between the operation members. In the second section of the paper the developed methodology is presented. The application of the approach to a dynamic logistics problem is described in the third section. Some results are summarised in the Conclusion.

\section{Proposed Approach}

The main idea of the approach is to represent business network members by sets of services provided by them Fig. 1. This makes it possible to replace the interoperability between business network members with that between their services.

At the first stage of the research the lifecycle phases of the service-based network and major requirements to them were defined (Table 1). Based on these requirements the main ideas the approach is based on were formulated:

1. A common shared top-level ontology (application ontology) serves for terminology unification. Each service has a fragment of this ontology corresponding to its capabilities / responsibilities. This fragment is synchronized automatically when necessary (not during the operation).

2. Each service has a profile describing its capabilities, appropriate ontological model.

3. Each service is assigned an intelligent agent, representing it (together they will be called "agent-based service"). The agent collects information required for situational understanding by the service, negotiates with other agents to create ad-hoc action plans. The agent has predefined rules to be followed during negotiation processes. These rules depend on the role of the appropriate member.

4. Web-service standards are used for interactions. External sources (e.g., medical databases, transport availability, weather forecasts) should also support these standards and the terminology defined by the application ontology. This is achieved by developing services for each particular source.

The developed methodology proposes a two-level framework of context-driven information integration for decision making. The first level addresses activities over a prestarting procedure of the system as creation of semantic models for its components (Fig. 2); accumulating domain knowledge; linking domain knowledge with the information sources; creation of an application ontology describing a macro-situation; indexing 


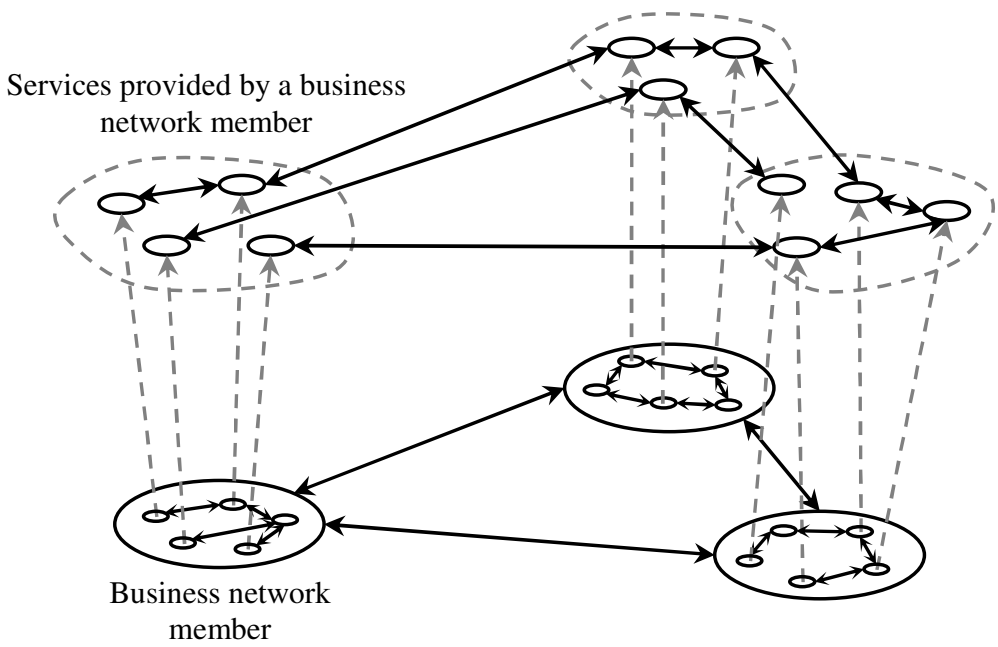

Fig. 1. Representation of business network members by sets of services

Table 1. Lifecycle phases for the service network, its needs and services to fulfil them

\begin{tabular}{lll}
\hline Life cycle phase & Needs & Services \\
\hline $\begin{array}{l}\text { Community building } \\
\text { (once, new members are } \\
\text { added on a continuous } \\
\text { basis) }\end{array}$ & Common infrastructure & $\begin{array}{l}\text { Modelling goals and } \\
\text { objectives }\end{array}$ \\
& $\begin{array}{l}\text { Common communication } \\
\text { standards and protocols }\end{array}$ & $\begin{array}{l}\text { Identification, qualification, } \\
\text { registration of members } \\
\text { Common knowledge } \\
\text { representation } \\
\text { Common modelling for } \\
\text { community members } \\
\text { Task modelling }\end{array}$ \\
$\begin{array}{l}\text { Formation (continuous, } \\
\text { initiated by the situation, } \\
\text { or a task as a part of the } \\
\text { situation) }\end{array}$ & $\begin{array}{l}\text { Task definition model (context) } \\
\text { Operation (continuous) }\end{array}$ & $\begin{array}{l}\text { Coordination and } \\
\text { Rules of partner selection }\end{array}$ \\
$\begin{array}{l}\text { Discontinuation } \\
\text { (continuous, initiated by } \\
\text { members) }\end{array}$ & $\begin{array}{l}\text { Termination of the established } \\
\text { agreements }\end{array}$ & $\begin{array}{l}\text { Rules of re-negotiation and } \\
\text { solution modification if } \\
\text { necessary } \\
\text { Update of the current } \\
\text { solution }\end{array}$ \\
\hline
\end{tabular}

a set of available e-documents against the application ontology. This level is supported, if required, by the subject experts, knowledge and ontology engineers.

The second level focuses on decision making supported by the system. This level addresses a problem recognition presented by a user request; context creation; identification of relevant knowledge sources; generation of a set of problem solutions; and making a decision by the user.

The internal knowledge representation is supported by the formalism of object-oriented constraint networks (OOCN) [21]. All the system components and contexts are represented 
System Components

Model

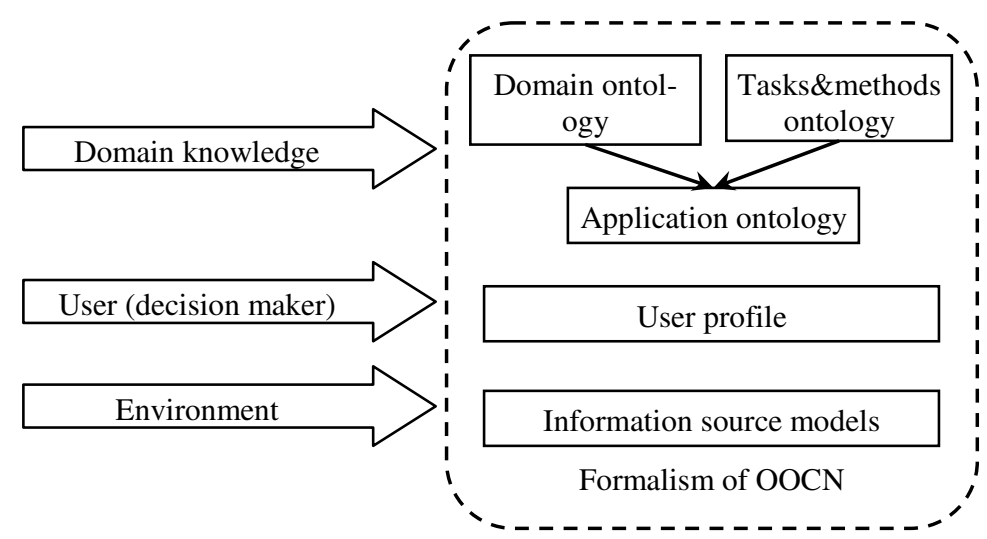

Fig. 2. Models for system components

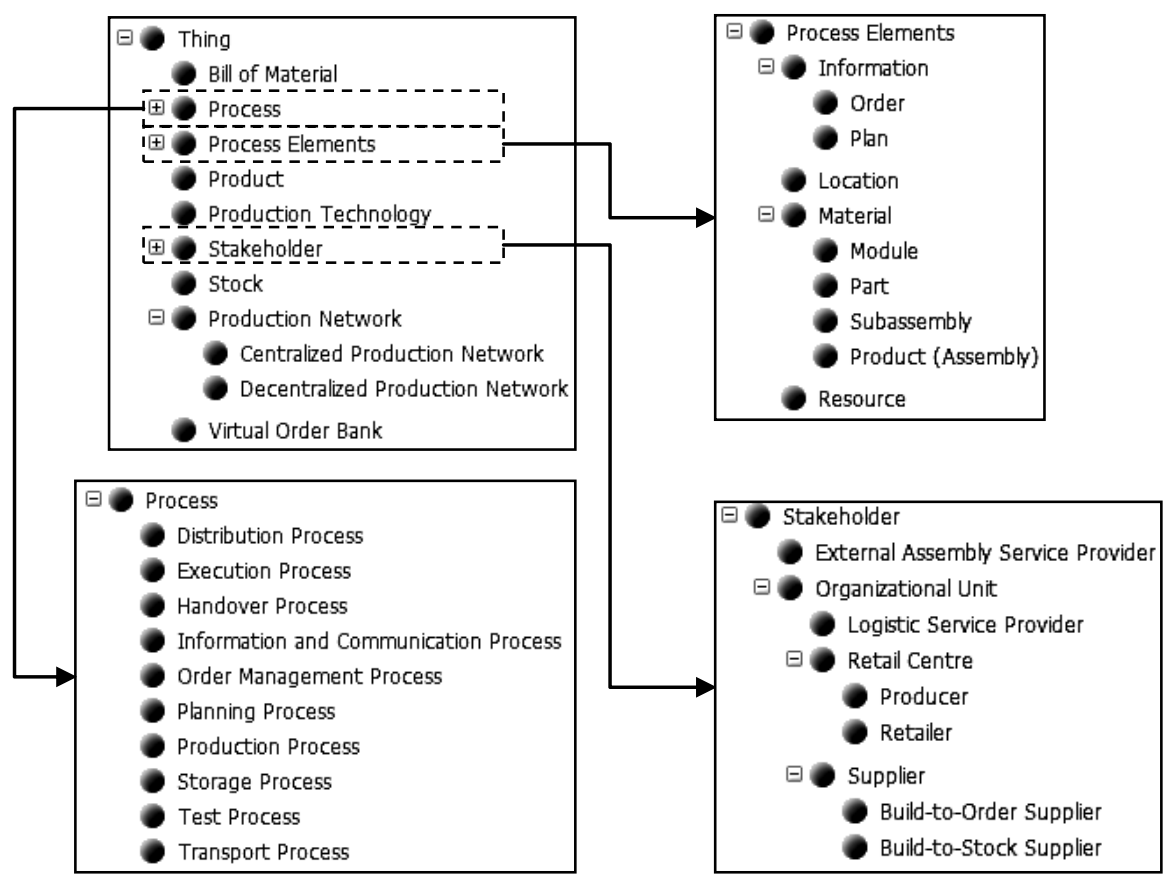

Fig. 3. Taxonomy of the application ontology for a production network

by means of this formalism. According to the formalism, the application ontology is represented as sets of classes, class attributes, attribute domains, and constraints. The set of constrains comprises constraints describing "class, attribute, domain" relation; constraints representing structural relations as hierarchical relationships "part-of" and "is-a", classes compatibility, associative relationships, class cardinality restrictions; and constraints describing functional dependencies. Below examples of some constraints are given: 
- "class, attribute, domain" relation: the attribute costs belongs to the class component and takes positive values;

- hierarchical relationship "is-a": the body production facility is a resource;

- hierarchical relationships "part-of": an instance of the class component can be a part of an instance of the class product;

- associative relationship: an instance of the class body can be connected to an instance of the class body production facility;

- classes compatibility: the class body is compatible with the class body production facility;

- functional dependence: the value of the attribute cost of an instance of the class body production facility depends on values of the attribute cost of instances of the class component connected to it and on the number of such instances.

Fig. 3 represents the macro-level taxonomy of the built by domain experts application ontology for a production network as an example of business network. The represented classes are the main concepts for the production network configuration problem.

To demonstrate implementation of the approach in a prototype related to the "Transportation Network Configuration" task is considered. In the case study the knowledge acquired from a number of sources is shared in order to find a feasible solution.

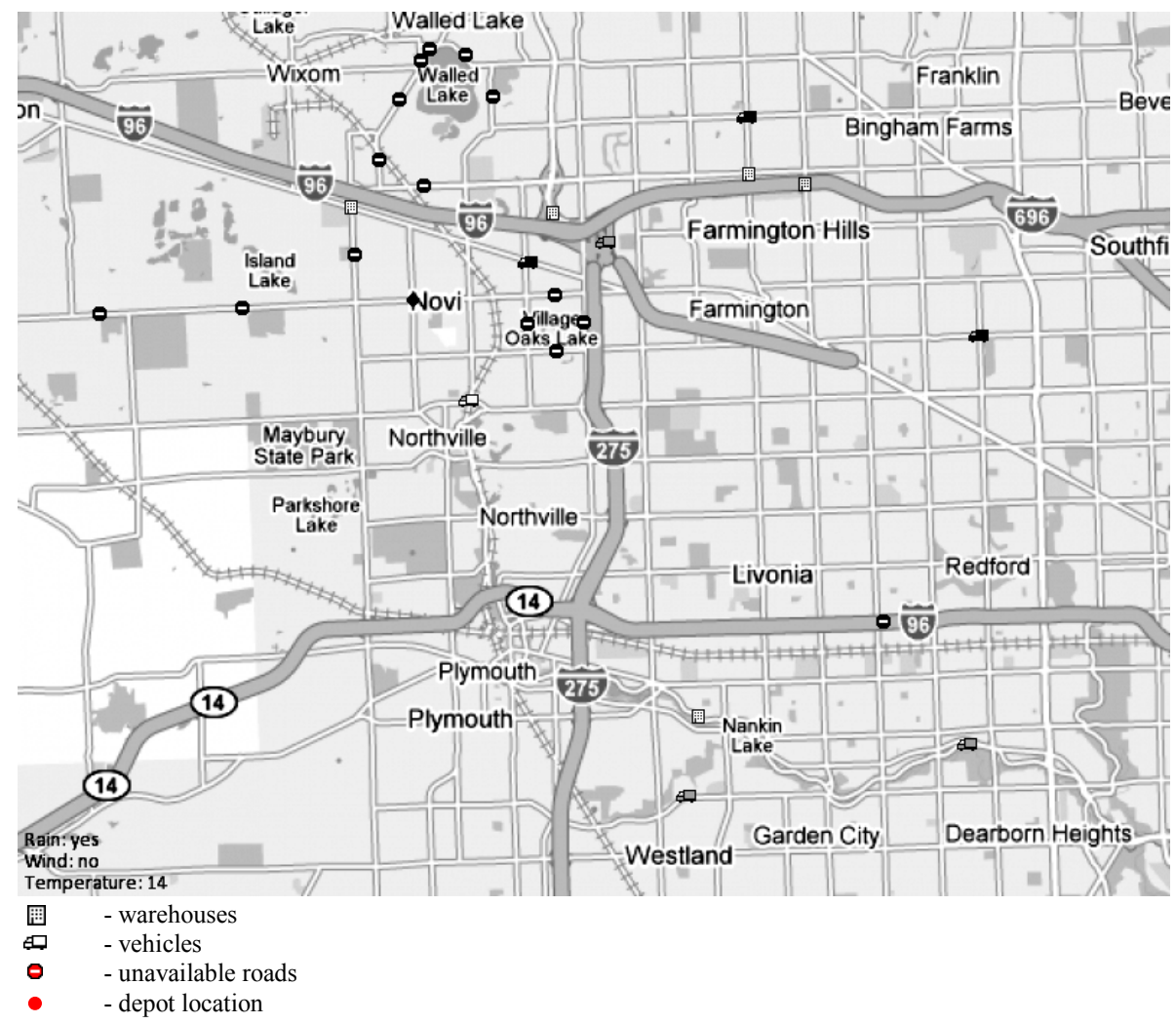

Fig. 4. Current situation for the dynamic transportation problem 


\section{Case Study}

Logistics management often involves such problems as creation of routing plans for a given set of vehicles available and goods to be transported. This is quite a common problem that has a number of solving techniques, e.g., [22]. However, in real life situations it is often necessary to take into account continuously changing traffic situation (e.g., to take into account traffic jams, closed roads, etc.) what makes the problem more complex and requires its solving it in real-time. This section presents application of the above described approach to this problem in the form of a Decision Support System (DSS).

In the case study the problem is considered as a dynamic transportation problem. There are several vehicles in different known locations that change in time. There is a central depot with a number of products to be transported (for simplicity containers will be considered instead of products and truck capacity will be one container). Also there exist several warehouses with given capacities where the products (containers) are to be transported. It is necessary to take into account the current situation in the area (traffic jams, closed roads, etc.) to find a feasible solution with possibly minimal time of transportation (Fig. 4). Though the problem seems to be simple it appears to be more complicated than it looks. For example, it can be more reasonable for one vehicle to make two or more rides instead of using two or more vehicles.

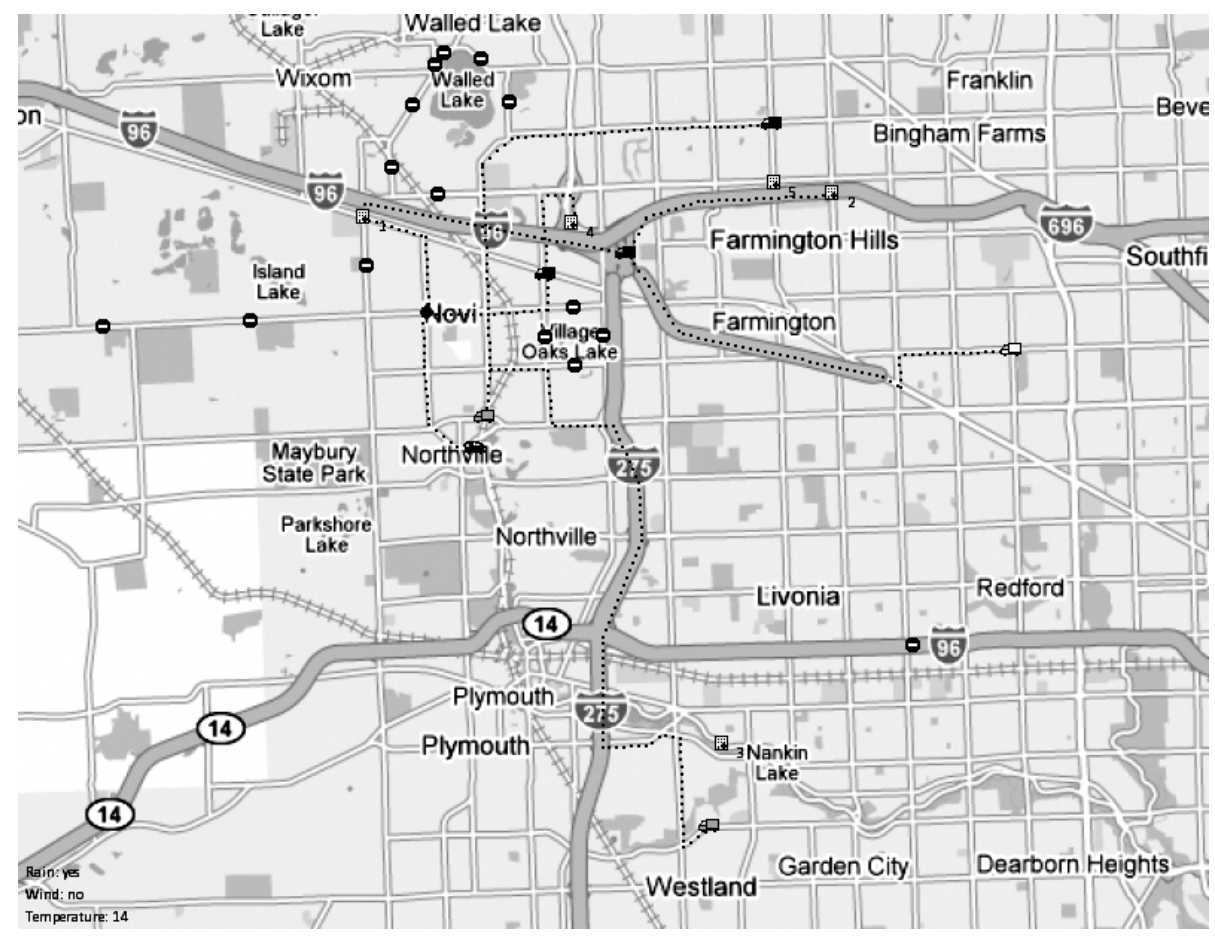

Fig. 5. Example of the solution for the dynamic transportation problem 


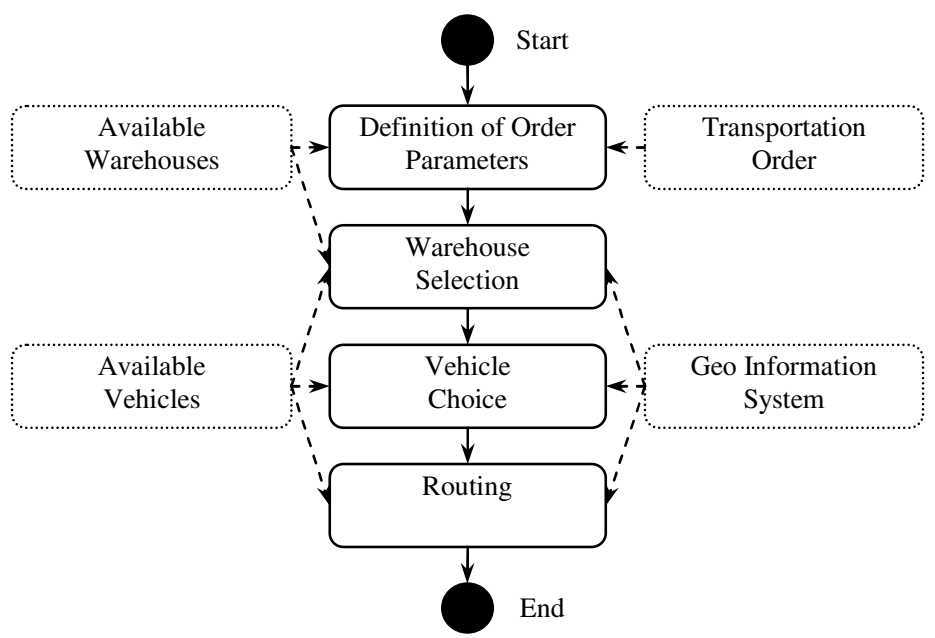

Fig. 6. Sequence of subtasks solving and information sources used

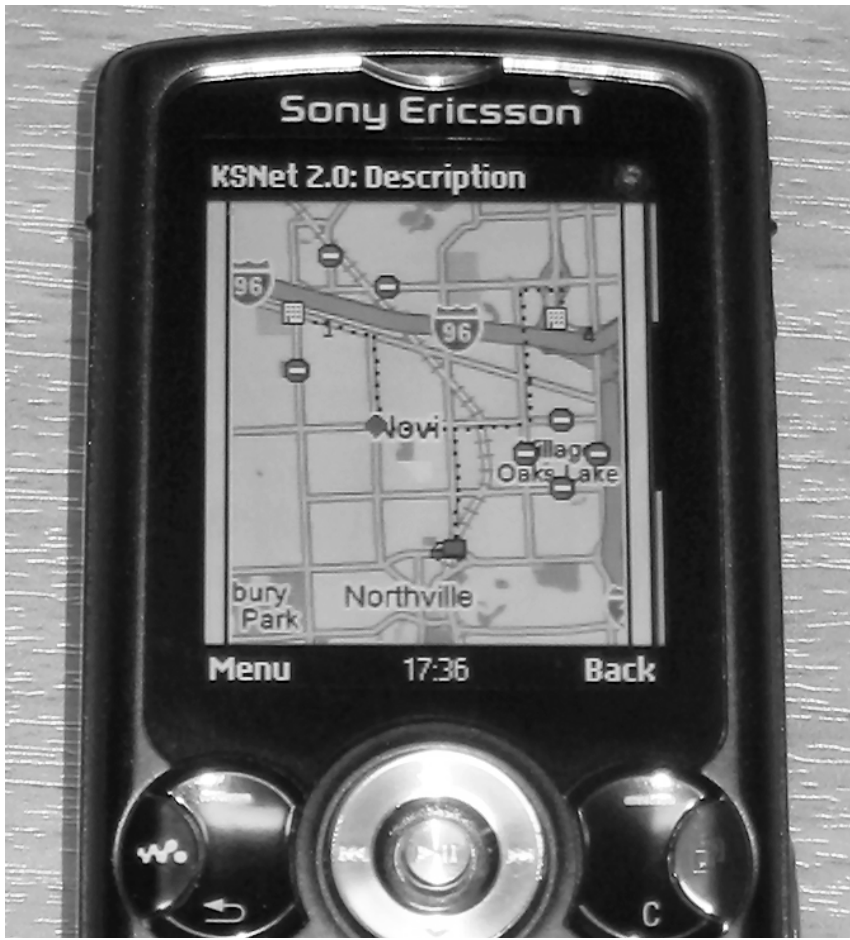

Fig. 7. Example of the vehicle driver assignment on the screen of a mobile phone 
An example of generated solution can be seen in Fig. 5 .

The problem solving methods have been implemented as a part of a distributed service network. Since information sources are distributed and heterogeneous, Webservices (called wrapper Web-services) have been used for organizing a unified access to them. The proposed architecture makes it possible to use problem solving software modules/services (e.g., the module implementing the described above algorithm) in the same way the sources are used.

Fig. 6 represents a diagram of case study services representing tasks to be solved and information sources used for their solving. Solid rectangles are tasks solved in a sequence. To solve the stated tasks information from heterogeneous sources (dotted rectangles) is used (information flows are indicated by dashed arrows).

The interface of the system is Web-based. This means that regular Web browsers can be used for working with the system. The decision maker can see an interactive map (Fig. 4, Fig. 5) and choose different parameters and criteria for problem solving. The drivers of the vehicles receive their assignments via Internet as well. They can see their routes using PDA or mobile phones (Fig. 7). Presented in the figures example includes 9 containers and 7 vehicles.

\section{Conclusions}

The paper represents an approach to service-based modeling of business networks. It is proposed that such representation can resolve problems arising from failures of centralized control. Ontologies have been proposed for description of problem domain knowledge. In accordance with the selected model of interoperability, services directly connect and exchange information with one another, but service descriptions (in the paper this is description of the knowledge source content and tasks that can be solved) are mapped to the common shared application ontology. As an example of the approach application the paper presents experience of solving a complex real-life problem from the area of dynamic logistics. The problem considered takes into account continuously changing environment and requires nearly real-time solving.

The further research activities will address development of algorithms for building self-organising service networks and generation and estimation of alternative network configurations. The authors believe that once completed the proposed architecture could efficiently work for a range of the real world problems.

\section{Acknowledgments}

The research described in this paper is supported by grants \# 06-07-89242 and \# 0807-00264 of the Russian Foundation for Basic Research; projects funded by grants \# 16.2.35 of the research program "Mathematical Modelling and Intelligent Systems", and \# 1.9 of the research program "Fundamental Basics of Information Technologies and Computer Systems" of the Russian Academy of Sciences (RAS) and project of the scientific program of St.Petersburg Scientific Centre of RAS. 


\section{References}

1. Gunasekaran, A., Lai, K., Cheng, T.: Responsive supply chain: a competitive strategy in a networked economy. Omega 36, 549-564 (2008)

2. Gunasekaran, A., Ngai, N.: Build-to-order supply chain management: literature review and framework for development. Journal of Operations Management 23(5), 423-451 (2005)

3. Christopher, M., Towill, D.: An integrated model for the design of agile supply chains. International Journal of Physical Distribution and Operations Management 31, 235-244 (2001)

4. Sen, W., Shin, Y.W., Kumar, A., Piplani, R.: Supply chain demand response strategy evaluation and optimization. In: Proceedings of the 2000 IEEE International Conference on Management of Innovation and Technology (ICMIT 2000), vol. 2, pp. 782-787 (2000)

5. SOA: Service-oriented architecture definition (2007),

http: / / www. service-architecture.com/

web-services/articles/service-

oriented_architecture_soa_definition.html

6. Web Services explained (2007), http://www.service-architecture.com/ web-services/articles/web_services_explained.html

7. Semantic Web (2006). Web site, http: / / www . semanticweb. org

8. Foundation for Intelligent Physical Agents (FIPA) Documentation, http: / / www. fipa.org

9. Heflin, J., Hendler, J.: Semantic Interoperability on the Web. In: Proceedings of Extreme Markup Languages 2000, pp. 111-120. Graphic Communications Association (2000)

10. Boury-Brisset, A.-C.: Ontology-Based Approach for Information Fusion. In: Proceedings of the Workshop on Ontology and Information Fusion of U.S Army CECOM \& the Center for Multisource Information Fusion (2003), http://www. infofusion.buffalo. edu/conferences_and_workshops/ontology_wkshop_2/ont_ws2_ working_materials/BouryBrissetontologyandFusion. PDF

11. Smirnov, A., Pashkin, M., Chilov, N., Levashova, T.: Knowledge Logistics in Information Grid Environment. International Journal on Future Generation Computer Systems (2004); Zhuge, H. (ed.): The special issue Semantic Grid and Knowledge Grid: The NextGeneration Web, vol. 20(1), pp. 61-79

12. Ruggaber, R.: Interoperability of Enterprise Systems and Applications. Strengthening Competitiveness through Production Networks. In: A perspective from European ICT research projects in the field of Enterprise Networking, pp. 58-70. European Communities (2005)

13. Viana, A.C., Amorim, M.D., Fdida, S., Rezende, J.F.: Self-organization in spontaneous networks: the approach of DHT-based routing protocols. Ad Hoc Networks J., special issue on Data Communications and Topology Control in Ad Hoc Networks 3(5), 589-606 (2005)

14. Hammer, B., Micheli, A., Sperduti, A., Strickert, M.: Recursive self-organizing network models. Neural Networks 17(8-9), 1061-1085 (2004)

15. Nakano, T., Suda, T.: Self-Organizing Network Services with Evolutionary Adaptation. IEEE Transactions on Neural Networks 16(5) (2005)

16. Chandran, R., Hexmoor, H.: Delegation Protocols Founded on Trust. In: KIMAS 2007: Modeling, Exploration, and Engineering (Proceedings of the 2007 International Conference on Integration of Knowledge Intensive Multi-Agent Systems), pp. 328-335. IEEE, Los Alamitos (2007) 
17. Baumgarten, M., Bicocchi, N., Curran, K., Mamei, M., Mulvenna, M., Nugent, C., Zambonelli, F.: Towards Self-Organizing Knowledge Networks for Smart World Infrastructures. In: Teanfield, H. (ed.) International Transactions on Systems Science and Applications, vol. 2(2), pp. 123-133 (2006) ISSN 1751-1461

18. Smirnov, A., Shilov, N., Kashevnik, A.: Constraint-Driven Negotiation Based on Semantic Interoperability in BTO Production Networks. In: Panetto, H., Boudjlida, N. (eds.) Interoperability for Enterprise Software and Applications (Proceedings of the Workshops and the Doctoral Symposium of the Second IFAC/IFIP I-ESA International Conference: EI2N, WSI, IS-TSPQ 2006), pp. 175-186. ISTE Ltd. (2006) ISBN-13 978-1-905209-61-3, ISBN-10 1-905209-61-4

19. Dey, A.K., Salber, D., Abowd, G.D.: A Conceptual Framework and a Toolkit for Supporting the Rapid Prototyping of Context-Aware Applications, Context-Aware Computing. In: Moran, T.P., Dourish, P. (eds.) A Special Triple Issue of Human-Computer Interaction, vol. 16. Lawrence-Erlbaum, Mahwah (2001)

20. Smirnov, A., Pashkin, M., Levashova, T., Chilov, N.: Fusion-Based Knowledge Logistics for Intelligent Decision Support in Network-Centric Environment. George J 34(6), 673690 (2005)

21. Smirnov, A., Sheremetov, L., Chilov, N., Sanchez-Sanchez, C.: Agent-Based Technological Framework for Dynamic Configuration of a Cooperative Supply Chain. Multiagentbased supply chain management. In: Chaib-draa, B., Müller, J.P. (eds.) Multiagent-based supply chain management. Series on Studies in Computational Intelligence, vol. 28, pp. 217-246. Springer, Heidelberg (2006)

22. Romero, M., Sheremetov, L., Soriano, A.: A Genetic Algorithm for the Pickup and Delivery Problem: an Application to the Helicopter Offshore Transportation. In: Castillo, O., Melin, P., Montiel-Ross, O., Sepúlveda-Cruz, R., Perdycz, W., Kacprzyk, J. (eds.) Theoretical Advances and Applications of Fuzzy Logic and Soft Computing, Advances in Soft Computing, vol. 42, pp. 435-444. Springer, Heidelberg (2007) 